\title{
NORMALLY CLOSED SATURATED FORMATIONS
}

\author{
MARK P. HALE, JR.
}

\begin{abstract}
Theorems of Gaschütz and Baer concerning the Frattini subgroup, the hypercenter, and nilpotent and supersolvable subgroups are extended to normally closed saturated formations.
\end{abstract}

The hypercenter, derived group, and Frattini subgroup are all related to nilpotency of a finite group. In [4], Gaschütz studied their relations, and found a sufficient condition for a normal subgroup of a group to be nilpotent. By replacing the notion of centrality with $\mathfrak{Y}$-centrality, we give theorems which extend the results of Gaschütz to normally closed saturated formations containing all nilpotent groups, and strengthen his nilpotency criterion. A theorem of Baer, on supersolvably immersed subgroups, is also extended to saturated formations.

In this paper, all groups are finite. A class of groups is understood to include, with $G$, all groups isomorphic to $G$. If $M$ is a subgroup of $G$, $\operatorname{core}(M)$ is the intersection of the $G$-conjugates to $M$, and is the largest normal subgroup of $G$ contained in $M$. A chief factor of $G$ is a quotient $H / K$, where $K \leqslant G$ and $H / K$ is minimal normal in $G / K$. If $H / K$ is a chief factor with $L \leqq K$ and $H \leqq M$, we say $H / K$ lies above $L$ and below $M$. A subgroup $M<G$ is a supplement to $H / K$ in $G$ if $M H=G$ and $M \cap H \geqq K$. If $M \cap H=K, M$ is a complement to $H / K$. Other notation and terminology is standard, and can be found in [6].

Although the results below concern classes of solvable groups, it is not assumed that all groups dealt with are solvable.

1. Let $\mathfrak{X}$ be a class of groups. A maximal subgroup $M$ of $G$ is $\mathfrak{X}$-normal if $G / \operatorname{core}(M)$ is in $\mathfrak{X}$, and $\mathfrak{X}$-abnormal otherwise. We define $T_{\mathfrak{X}}(G)$ to be the intersection of $G$ with all $\mathfrak{X}$-normal maximal subgroups of $G$. If $\mathfrak{X}$ is the class of all groups, $T_{\mathfrak{X}}(G)$ is the Frattini subgroup, $\Phi(G)$. Many useful properties of $\Phi(G)$ apply to $T_{\mathfrak{X}}(G)$; we collect them here.

Received by the editors March 29, 1971.

AMS 1970 subject classifications. Primary 20D10, 20D25, $20 \mathrm{D} 35$.

Key words and phrases. Saturated formation, Fratini subgroup, hypercenter, immersion.

(c) American Mathematical Society 1972 
Proposition 1. Let $\mathfrak{X}$ be an arbitrary class of groups.

(a) $T_{\mathfrak{X}}(G)$ is characteristic in $G$.

(b) If $N \leqslant G, N \leqq T_{\mathfrak{X}}(G)$, then $T_{\mathfrak{X}}(G / N)=T_{\mathfrak{X}}(G) / N$.

(c) If $N$ is minimal normal in $G$, then $N \leqq T_{\mathfrak{X}}(G)$ if and only if $N$ is not supplemented by an $\mathfrak{X}$-normal maximal subgroup of $G$.

(d) For any homomorphism $\theta$ of $G, T_{\mathfrak{X}}(G \theta) \geqq T_{\mathfrak{X}}(G) \theta$.

Proof. For automorphisms $\theta$ of $G, M \theta$ is $\mathfrak{X}$-normal if $M$ is; (a) follows. For (b), we observe that if $N \leqq M, M$ is $\mathfrak{X}$-normal in $G$ if and only if $M / N$ is $\mathfrak{X}$-normal in $G / N$. A maximal subgroup $M$ supplements a minimal normal subgroup $N$ if and only if it does not contain it; this proves (c). Since pre-images of $\mathfrak{X}$-normal maximal subgroups are $\mathfrak{X}$-normal, the preimage of $T_{\mathfrak{X}}(G \theta)$ contains $T_{\mathfrak{X}}(G)$, proving (d) and the proposition.

The third part of the lemma above yields the familiar fact that a chief factor $M / N$ has a supplement in $G / N$ if and only if $M ! N$ is not a Frattinifactor, i.e. is not contained in $\Phi(G / N)$. We will need the next lemma in later sections.

LEMMA 2. If the chief factor $H / K$ is supplemented by a maximal subgroup $M$ of $G$ and $G / \operatorname{core}(M)$ is solvable, then $H / K$ is Abelian, and $M$ complements $H / K$.

Proof. We may assume that $K=1$ and $H$ is minimal normal in $G$. Let $L=\operatorname{core}(M)$. Since $G / L$ is solvable and $H L / L \neq 1$, we have $H^{\prime}<H$ and so $H^{\prime}=1$. Now $H \cap M$ is normal in $M$ and centralized by $H$, so $H \cap M$ is normal in $G=H M$; thus $H \cap M=1$.

2. A nonempty class $\tilde{\Im}$ of solvable groups is a formation if:

(1) $\mathfrak{F}$ contains all homomorphic images of groups in $\mathfrak{F}$, and

(2) if $G / M$ and $G / N$ are in $\tilde{F}, G /(M \cap N)$ is in $\widetilde{F}$.

Each group $G$ has a smallest normal subgroup $G_{\mathfrak{J}}$ such that $G / G_{\tilde{\mho}}$ is in $\tilde{F}$. If $\mathfrak{F}$ contains all normal subgroups of groups in $\tilde{F}$, we say $\mathfrak{F}$ is normally closed. A formation is saturated if $G / \Phi(G)$ in $\widetilde{F}$ implies $G$ belongs to $\widetilde{F}$. All saturated formations can be constructed by the following recipe, first given in [5]. For each prime $p$, a formation $f(p)$ is specified. The class $\tilde{f}(f)$ of all solvable groups $G$ with $G / O_{p^{\prime}, p}(G) \in f(p)$ for each $p$ is a saturated formation, locally defined by $\{f(p)\}$. If it is required that $f(p) \subseteq \mathfrak{F}(f)$, and that $f(p)$ contain each group $G$ for which $G / O_{p}(G) \in f(p)$, then there is a unique choice for the formations $\{f(p)\}$. When we speak below of a saturated formation, it is this choice of the formations $\{f(p)\}$ to which we refer. This background material is to be found in [2] and [8], or [3].

A chief factor $H / K$ of $p$-power order is $\widetilde{\mho}$-central if Aut $_{G}(H / K)=$ $G / C_{G}(H / K)$ lies in $f(p)$. A group $G$ belongs to a saturated formation $\tilde{\mho}$ if and only if each supplemented factor of $G$ is $\tilde{i}$-central. The next lemma 
shows that our definition of $\widetilde{\mho}$-normal coincides with that given for saturated formations in [2].

Proposition 3. Let $\widetilde{\mho}$ be a saturated formation. A maximal subgroup $M$ of $G$ is $\mathfrak{F}$-normal if and only if $M$ complements an $\mathfrak{F}$-central chief factor of $G$.

Proof. Let $J=\operatorname{core}(M)$. If $M$ is $\mathfrak{F}$-normal, and $K / J$ is minimal normal in $G / J, M$ complements $K / J$, and since $G / J \in \tilde{F}, K / J$ is $\widetilde{\mho}$-central. Conversely, suppose $M H=G, M \cap H=K, H / K$ is minimal normal in $G / K$, and $H / K$ is $\widetilde{f}$-central. Then $L=C_{G}(H / K) \geqq H$, so $L=L \cap M H=(L \cap M) H$. Since $L \cap M \mid K$ is normal in $G / K, L \cap M$ is normal in $G$. Thus $L \cap M \leqq J$. On the other hand, $[H, J] \leqq H \cap M=K$, so $H J=C_{G}(H \mid K)$. Thus $A_{4 t}(H \mid K)=$ $G / H J$ belongs to $f(p) \subseteq \mathfrak{F}$, and since $H J / J \cong H / H \cap J=H / K, H J / J$ is $\mathfrak{F}$ central, so $G / J \in \mathfrak{F}$.

For $\mathfrak{F}$ a saturated formation, we define the $\mathfrak{F}$-Frattini subgroup $\Phi_{\mathfrak{q}}(G)$, to be the subgroup $T_{\mathfrak{F}^{\prime}}(G)$, where $\widetilde{F}^{\prime}$ is the class of all groups not in $\widetilde{F}$. We note that $\Phi_{\mathfrak{Y}}(G) \cap T_{\mathfrak{F}}(G)=\Phi(G)$; also by (c) of Proposition 1 and Lemma 2 , every supplemented chief factor below $\Phi_{\mathfrak{F}}(G)$ is complemented by an $\widetilde{\mho}$-normal maximal subgroup and is therefore $\mathfrak{F}$-central. We now give a strengthened test for saturation.

THEOREM A. The formation $\mathfrak{F}$ is saturated if and only if $G / \Phi_{\mathfrak{F}}(G) \in \mathfrak{F}$ implies $G \in \mathbb{F}$.

Proof. If $\mathscr{F}$ is saturated, and $G / \Phi_{\mathfrak{r}}(G) \in \mathfrak{F}$, then as remarked above, each supplemented chief factor of $G / \Phi(G)$ is $\mathfrak{F}$-central, and $G / \Phi(G) \in \mathfrak{F}$, so $G \in \tilde{\mho}$. Conversely, since $\Phi_{\mathfrak{r}}(G) \geqq \Phi(G), G / \Phi(G) \in \mathfrak{F}$ implies $G / \Phi_{\mathfrak{F}}(G) \in \tilde{\mho}$, so $G \in \mathbb{F}$ if the condition holds.

3. Throughout this section, $\mathbb{F}$ will denote a saturated formation. A subgroup $H$ of $G$ is said to be $\mathfrak{F}$-immersed in $G$ if $H \leqslant G$ and each $G$-chief factor below $H$ is $\tilde{i}$-central. The $\mathfrak{F}$-hypercenter, $Z_{\mathfrak{F}}(G)$, is defined to be the product in $G$ of all $\mathfrak{F}$-immersed subgroups of $G ; Z_{\mathfrak{F}}(G)$ is $\tilde{F}$-immersed, and characteristic in $G$. In the language of [2], $Z_{\mathfrak{F}}(G)$ is the intersection of the $\tilde{y}$-normalizers of $G$, if $G$ is solvable.

PROPOSITION 4. For a saturated formation $\mathfrak{F}, Z_{\mathfrak{F}}(G) \leqq \Phi_{\mathfrak{F}}(G)$.

Proof. Let $N$ be a minimal normal subgroup of $G$, contained in $Z_{\mathfrak{F}}(G)$. Then $Z_{\mathfrak{F}}(G / N)=Z_{\mathfrak{F}}(G) / N$. If $N$ is not contained in $\Phi_{\mathfrak{F}}(G), N$ is complemented by an $₹$-abnormal maximal subgroup, so $N$ is not $\mathbb{F}$-central, a contradiction. Thus $N \leqq \Phi_{\mathfrak{F}}(G)$, and $\Phi_{\mathfrak{F}}(G / N)=\Phi_{\mathfrak{F}}(G) / N$. By induction, $Z_{\mathfrak{F}}(G) / N \leqq \Phi_{\mathfrak{F}}(G) / N$, completing our proof.

COROLlaRY 5. $Z_{\mathfrak{F}}(G) \cap G_{\xi} \leqq \Phi(G)$.

PROOF. $\quad Z_{\mathfrak{F}}(G) \cap G_{\mathfrak{F}} \leqq \Phi_{\mathfrak{F}}(G) \cap T_{\mathfrak{F}}(G)=\Phi(G)$. 
RemarK. The theorem of Gaschütz [4] which Corollary 5 emulates is somewhat sharper, reading $Z_{\infty}(G) \cap G^{\prime} \leqq \Phi(G)$. We can recover this by observing that if $\tilde{F}^{*}$ is the smallest saturated formation containing $\tilde{F}$, then one can show that $T_{\tilde{\gamma}}(G)=T_{\tilde{\gamma}^{*}}(G)$, so that $Z_{\tilde{r} *}(G) \cap G_{\tilde{\aleph}} \leqq \Phi(G)$, by the proof given above.

It is reasonable to ask whether or not $\tilde{i}$-immersed subgroups are actually in $F$.

Proposition 6. For a saturated formation $\tilde{x}$, $\tilde{\mho}$-immersed subgroups lie in $\tilde{F}$ if and only if $\tilde{\mho}$ is normally closed.

Proof. If $H \leqslant G \in \tilde{\mathscr{y}}$, and $\tilde{y}$-immersed subgroups lie in $\widetilde{\mho}, H \in \tilde{\mathscr{y}}$. Conversely, suppose $H$ is $\tilde{F}$-immersed in $G$, and $\mathfrak{F}$ is normally closed. By Lemma 5.2 of [3], each $f(p)$ is normally closed. Now if $M / N$ is a $G$ chief factor below $H$, Aut $_{H}(M / N)$ is isomorphic to a normal subgroup of Aut $_{i}(M / N)$, so $\operatorname{Aut}_{H}(M / N)$ belongs to $f(p)$. If $K \S H$ and $N \leqq K \leqq M$, then $C_{H}(K / N)$ and $C_{H}(M / K)$ contain $C_{H}(M / N)$, so $\operatorname{Aut}_{H}(K / N)$ and Aut $_{H}(M / K)$ are quotients of $\mathrm{Aut}_{H}(M / N)$ and are in $f(p)$. Thus if we refine the $G$-chief factors below $H$ to $H$-chief factors they remain $\mathfrak{f}$-central, and thus $H \in \tilde{F}$.

The following technical result is the basis of our study of $\mathfrak{F}$-immersed subgroups.

THEOREM B. Let $H$ be a solvable normal subgroup of an arbitrary finite group $G$, and let $p$ be a prime. For any subgroup $C$ of $G$, if $C$ centralizes each p-chief factor of $G$ between $H$ and $\Phi(H), C$ centralizes each p-chief factor of $G$ below $\Phi(H)$.

Proof. We may assume that $C$ is the intersection of the centralizers of the $p$-chief factors between $H$ and $\Phi(H)$, so that $C \leqslant G$. By a routine induction argument, it is sufficient to consider the following case: $K$ is a minimal normal $p$-subgroup of $G$ contained in $\Phi(H)$, and $C$ centralizes all $p$-factors of $G$ between $K$ and $H$.

For any prime $r$ dividing $|C|$, let $R$ be an $r$-Sylow subgroup of $C$. Define $S=H R$ and $T=C \cap S ; S$ is solvable, and $R \leqq T \leqq S$. Now set $N=N_{S}(T \cap U)$, where $U$ is a $p^{\prime}$-Hall subgroup of $S$. Since $T$ normalizes $H$, $H$ can be regarded as a group with operators, the conjugations from $T$. Let $A / B$ be a $T$-chief factor of $H$. Since $U \leqq N, N B \geqq A$ if $A / B$ is not a $p$-group. If $A / B$ is a $p$-group, then $\mathrm{P}$. Hall's theory of system normalizers [7] applies. This yields that $A \leqq N B$ if $T$ centralizes $A / B$, and $A \cap N \leqq B$ otherwise. Since $T \leqq C$ centralizes all $T$-factors of $p$-power order above $K$, $N K \cap H=H=K(N \cap H)=\Phi(H)(N \cap H)=N \cap H$. Thus $H \leqq N$, and $T$ centralizes all $T$-factors of $H$ contained in $K$. Thus for some sufficiently long commutator, $[K, R, \cdots, R]=1$. For $r \neq p$, we have $[K, R]=1$ by 
Theorem 5.3.2 of [6]. Therefore $C_{G}(K)$ contains the $r$-Sylow subgroups of $C$ for all primes $r \neq p$. Thus for any $p$-Sylow subgroup $P$ of $C, C \leqq P C_{G}(K)$. Now we have $[K, C] \leqq\left[K, P C_{G i}(K)\right]=[K, P]<K$. Since $C$ is normal and $K$ is minimal normal, we have $[K, C]=1$, completing our proof.

Our next theorem extends the main result of [1].

THEOREM C. Let is be a saturated formation. If $H$ is a solvable normal subgroup of an arbitrary finite group $G$ and $H / \Phi(H)$ is $\mathfrak{F}$-immersed in $G / \Phi(H)$, then $H$ is $\tilde{y}$-immersed in $G$.

Proof. By induction, we need only study the case where $M$ is minimal normal in $G, M \leqq \Phi(H)$, and $H / M$ is $\tilde{s}$-immersed in $G / M$. Since $M \leqq \Phi(H)$, $M$ is a $p$-group for some prime $p$. Let $C$ be the intersection of the centralizers of the $p$-chief factors of $G$ between $M$ and $H$. Since these factors are each $\widetilde{\mho}$-central, $G / C \in f(p)$. By Theorem B, $C$ centralizes $M$, so $\operatorname{Aut}_{G}(M)$ is a quotient of $G / C$ and therefore also lies in $f(p)$. By definition, $M$ is an $\tilde{F}^{-}$ central factor, and $H$ is $\bar{j}$-immersed as claimed.

For the formation of nilpotent groups, i্s-central factors (alias central factors) can be detected by use of commutators; we now generalize this to other saturated formations. For $H \leqslant G$, define the $\mathfrak{\mho}$-hypocenter of $H$ relative to $G$ to be the smallest $G$-normal subgroup $K$ contained in $H$ so that all $G$-chief factors between $K$ and $H$ are $\mathfrak{F}$-central; we write $[H, G ; \mathfrak{F}]$ for this subgroup.

PROPOSITION 7. Let $\tilde{y}$ be a saturated formation.

(a) $A$ G-chief factor $H / K$ is $\tilde{\mho}$-central if $[H, G ; \widetilde{\mho}] \leqq K$.

(b) $[G, G ; \mathfrak{F}]=G_{\mathfrak{x}}$.

(c) If $\mathfrak{F}$ is normally closed and $H \leqslant G$, then $[H, G ; \mathfrak{F}] \leqq G_{\mathfrak{F}} \cap H$.

Proof. The first two parts are trivial; for (c) we note that the $G$-chief factors from $G_{\tilde{y}} \cap H$ to $H$ are $G$-isomorphic to those from $H G_{\mathfrak{\mho}}$ to $H G_{\tilde{\S}} / G_{\mathfrak{⿰}}$, which are $\tilde{\mho}$-central with respect to $G$; apply Proposition 6.

We now give an analogue of a resuit on $\Phi$-free groups due to Gaschütz.

THEOREM D. Let ĩ be a saturated, normally closed formation. Then if $\Phi(G)=1, Z_{\tilde{\mathfrak{\gamma}}}(G)=\Phi_{\mathfrak{\gamma}}(G)$.

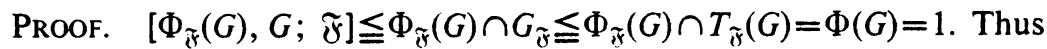
$\Phi_{\tilde{r}}(G)$ is $\tilde{\mho}$-hypercentral, so $Z_{\mathfrak{F}}(G)=\Phi_{\mathfrak{z}}(G)$.

4. Gaschütz showed in [5] that a saturated formation $\mathfrak{F}$ containing all nilpotent groups has the following property: for a normal subgroup $H$ of $G$, if $H /(H \cap \Phi(G))$ is in $\widetilde{\Im}$, then $H$ belongs to $\widetilde{F}$. It is easy to see that this property characterizes such formations. We now exhibit a stronger property which characterizes the saturated, normally closed formations containing the nilpotent groups. 
THEOREM E. For a formation $\mathfrak{F}$, the following properties are equivalent:

(a) $\mathfrak{F}$ is normally closed, saturated, and contains all nilpotent groups.

(b) If $H \preccurlyeq G$ and $H /\left(H \cap \Phi_{\mathfrak{\mho}}(G)\right)$ belongs to $\mathfrak{F}$, then $H$ belongs to $\mathfrak{F}$.

Proof. Assume (a), and suppose $N$ is a minimal normal subgroup of $G$ contained in $H \cap \Phi_{\tilde{y}}(G)$ and $H / N \in \widetilde{F}$. If $N \leqq \Phi(G), H \in \mathfrak{F}$ by the Theorem of Gaschütz cited above. If $N \lesseqgtr \Phi(G), N \cap \Phi(G)=1$, and by Theorem D, $N$ is $\mathfrak{F}$-central. By the proof of Proposition $6, N$ refines to $\mathfrak{F}$-central $H$ factors, and therefore $H \in \mathfrak{F}$.

Now assume (b); if $G \in \mathfrak{F}, G$ has no $\mathfrak{F}$-abnormal maximal subgroups, so $\Phi_{\mathfrak{r}}(G)=G$, and for all $H \triangleleft G, H / H \cap \Phi_{\mathfrak{F}}(G)=1$, so $H \in \mathfrak{F}$. With $H=G$, (b) becomes the condition for saturation given in Theorem A. The FFrattini subgroup of a cyclic group of order $p^{2}$ is at least order $p$, so $\mathfrak{F}$ contains all groups of prime order and therefore all nilpotent groups.

COROLLARY 8. If $\mathfrak{F}$ satisfies condition (a) of Theorem $\mathrm{E}, \Phi_{\mathfrak{F}}(G)$ belongs to $\mathfrak{5}$.

\section{BIBLIOGRAPHY}

1. R. Baer, Supersoluble immersion, Canad. J. Math. 11 (1959), 353-369. MR 29 \#4187.

2. R. W. Carter and T. O. Hawkes, The $\mathfrak{Y}$-normalizers of a finite soluble group, J. Algebra 5 (1967), 175-202. MR 34 \#5914.

3. B. Fischer, Classes of conjugate subgroups in finite solvable groups, Yale University, New Haven, Conn., 1966.

4. W. Gaschütz, Über die $\Phi$-Untergruppe endlicher Gruppen, Math. Z. 58 (1953), 160-170. MR 15, 285.

5. - Zur Theorie der endlichen auflösbaren Gruppen, Math. Z. 80 (1962/63), 300-305. MR 31 \#3505.

6. D. Gorenstein, Finite groups, Harper and Row, New York, 1968. MR 38 \#229.

7. P. Hall, System normalizers of a solvable group, Proc. London Math. Soc. (2) 43 (1937), 507-528.

8. B. Huppert, Endliche Gruppen. I, Die Grundlehren der math. Wissenschaften, Band 134, Springer-Verlag, Berlin and New York, 1967. MR 37 \#302. 32601

Department of Mathematics, University of Florida, Gainesville, Florida 